\title{
ANÁLISE DA CONCENTRAÇÃO ENERGÉTICA BRASILEIRA DAS INDÚSTRIAS DE CAL E GESSO, VIDREIRO E NÃO ENERGO-INTENSIVOS
}

\section{ANALYSIS OF THE BRAZILIAN ENERGY CONCENTRATION OF LIME AND PLASTER, GLASS AND NON-ENERGY-INTENSIVE INDUSTRIES}

\author{
Luiz Moreira Coelho Junior ${ }^{1}$ \\ Filipe Vanderlei Alencar ${ }^{2}$
}

Edvaldo Pereira Santos Júnior ${ }^{3}$

\begin{abstract}
Resumo: A Empresa de Pesquisa Energética (EPE) constitui o segmento de "Outras Indústrias" na matriz energética brasileira com os setores de cal e gesso, vidreiro e não energo-intensivos. Este artigo analisou a concentração energética do setor brasileiro de outras indústrias (SBOI), de 1970 a 2016. As informações foram extraídas do Balanço Energético Nacional (BEN) da EPE. A concentração energética foi determinada pelos indicadores: Razão de concentração [CR(p)], Índice de Hirschman - Herfindahl (HHI), Índice de Theil (E) e índice de Gini (G). Os resultados apresentaram que o consumo energético do SBOI teve um crescimento médio de 3,59\% a.a. de 1970 até 1982, o Óleo Combustível teve como principal fonte a energética e foi substituída pela eletricidade a partir de 1983, devido ao tamanho das firmas e a disponibilidade de outras fontes de energia. A demanda por gás natural (1995 a 2016) cresceu 5,66\% a.a., principalmente, em função dos incentivos no segmento vidreiro. O CR(p) mostrou-se altamente concentrado por meio dos valores médios de CR(1) de 47,69\%, CR(2) de 70,54\% e CR(4) de 91,51\%.O HHI inferiu como muito concentrado e o $E$ corroborou com essas análises. O G mostrou tendência de concentração com desigualdade médio a forte para muito forte. Conclui-se que o SBOI é concentrado na sua demanda energética.
\end{abstract}

Palavras-chave: Economia da energia; matriz energética; indicadores de concentração.

Abstract: The Energy Research Company (EPE) is the "Other Industries" segment in the Brazilian energy matrix with lime and gypsum, glass and non-energy-intensive sectors. This article analyzed the energy concentration of the Brazilian sector of other industries (SBOI) from 1970 to 2016. The information was extracted from the National Energy Balance (BEN) of the EPE. The energy concentration was determined by the following indicators: Concentration ratio $[C R(p)]$, Hirschman-Herfindahl index (HHI), Theil index (E) and Gini index (G). The results showed that SBOI energy consumption had an average growth of $3.59 \%$ per year. From 1970 to 1982, Fuel Oil was the main energy source, being replaced by electricity since 1983 , due to the size of the firms and the availability of other energy sources. The demand for natural gas (1995 to 2016) increased by $5.66 \%$ pa, mainly due to the incentives in the glass segment. CR(p) was highly concentrated by means of the mean values of CR(1) of $47.69 \%, C R(2)$ of $70.54 \%$ and $C R(4)$ of $91.51 \%$. HHI inferred as very concentrated and $E$ corroborated these analyses. $G$ showed a tendency of concentration from medium to strong to a very strong inequality. It is concluded that SBOI is concentrated in its energy demand.

Keywords: Energy economy; energy matrix; concentration indicators.

\section{INTRODUÇÂO}

De acordo com a Empresa de Pesquisa Energética - EPE (2014), o consumo

\footnotetext{
${ }^{1}$ Economista, Dr., Professor do Departamento de Engenharia de Energias Renováveis, Universidade Federal da Paraíba - UFPB, Brasil. E-mail: luiz@cear.ufpb.br.

2 Eng. de Energias Renováveis, Universidade Federal da Paraíba - UFPB, Brasil. E-mail: alencarfv@gmail.com.

${ }^{3}$ Eng. de Energias Renováveis, Mestrando em Energias Renováveis, Universidade Federal da Paraíba - UFPB, Brasil. E-mail: edvaldo.junior@cear.ufpb.br.
} 
energético das indústrias de Cal, Gesso e Vidro são classificados na matriz energética brasileira como "Outras Indústrias". Esses setores são bastantes diversificados nos seus processos produtivos e em relação à demanda de fontes de energia.

No Brasil, em 2014, o setor vidreiro apresentou déficit na balança comercial de US\$ 547,3 milhões e importações de US\$ 800,4 milhões sendo o vidro, o principal produto importado. Do faturamento mundial de 2011 para o segmento de vidros, o Brasil representou 5,3\% (US\$33,4 bilhões). O setor brasileiro de Cal, em 2013, produziu 543 milhões de toneladas e foi o $5^{\circ}$ no ranking mundial, gerando $R \$ 2,6$ bilhões, que atendeu basicamente o mercado interno (BAJAY; LEITE; GORLA, 2010a).

O setor brasileiro de outras indústrias (SBOI) tem sua importância no PIB, refletindo também na demanda energética. Segundo o Balanço Energético Nacional (BEN) de 1970 a 2016, o consumo no SBOI ocupou entre a $3^{\text {a }}$ e a $6^{a}$ do ranking industrial. Em 2016, O SBOI ficou em $4^{\circ}$ lugar e consumiu 7,9 milhões tep $(9,02 \%)$, sendo a Eletricidade $(50,72 \%)$, o Gás Natural (28,76\%) e a Lenha $(10,78 \%)$ responsáveis com cerca de $90 \%$ da oferta interna de energia deste segmento (EPE, 2017).

A eletricidade se tornou a principal fonte no SBOI devido aos ganhos produtividade. Santana e Bajay (2016) mostraram que a eficiência energética diminuiu o consumo de energia com a mesma produção, devido a evolução tecnológica, melhoria de processos e substituição das fontes de energia. Foram identificadas as potencialidades e previu-se uma queda de $20 \%$ da demanda energética para 2020 . A substituição foi uma marca inovadora no âmbito energético, porém, atualmente, a palavra de ordem é aproveitamento energético (EPE, 2014).

Para a identificação dos ganhos e que seja possível averiguar essas mudanças graduais do consumo energético (eficiência e intensidade) é necessária a utilização de índices de desempenho. Os indicadores de concentração são componentes importantes para medir estrutura de mercado, oferta e demanda em apenas um indicador (POSSAS, 1999, COELHO JUNIOR, 2013).

Os indicadores de concentração podem ser utilizados em diversas áreas do conhecimento, como na engenharia florestal com os ensaios de Coelho Junior, Reszende e Oliveira (2013), Coelho Junior (2016) e Simioni et al. (2017), na agropecuária com Costa e Santana (2014), Cuenca e Dompieri (2017) e Coelho Junior (2017), no bancário com Luft e Zilli (2013), na siderurgia com Oliveira e Sperb (2016). No setor energético com Beiral, Moraes e Bacchi (2013), Coelho Junior et al. (2017), Coelho Junior et al. (2018) e Coelho Junior, Santos Junior e Alencar (2019).

Em busca de compreender melhor o comportamento da demanda energética industrial, este artigo analisou a concentração energética do setor brasileiro de outras indústrias (SBOI), de 1970 a 2016. 


\section{MATERIAIS E MÉTODOS}

Os dados usados para medir a concentração energética do Setor Brasileiro de Outras Indústrias (SBOI) foram obtidos do Balanço Energético Nacional (BEN). A unidade de medida utilizada foi em tonelada equivalente de petróleo (tep), entre 1970 e 2016.

Analisou-se a conjuntura do consumo energético do SBOI nos anos de 1970, 1975, 1980, 1985, 1990, 1995, 2000, 2005, 2010 e 2016. Avaliaram-se os ganhos e perdas das fontes energéticas por meio da Taxa Geométrica de Crescimento (TGC), Equação 1 (CUENCA; DOMPIERI, 2017).

$$
T G C[\%]=\left[\Delta t \sqrt{\frac{V_{F}}{V_{O}}}-1\right] * 100
$$

em que, $V_{F}$ é o consumo energético referente ao ano final; $V_{O}$ é o consumo energético referente ao ano inicial; $\Delta t$ é a diferença entre o ano final e o inicial.

A concentração energética foi mensurada por meio de índices parciais (parte dos dados) e sumárias (totalidade de informações). O índice parcial usado foi a razão de concentração e os índices sumários utilizados foram o Índice de Hirschman-Herfindahl, o Índice de Entropia de Theil e o Índice de Gini.

A Razão de concentração [CR(p)], Equação2, foi proposto Bain (1959). Este indicador avaliou a parcela do consumo energético $p(i=1,2,3, \ldots, p)$ fontes da matriz energética do SBOI.

$$
C R(p)=\sum_{i=1}^{p} S_{i}
$$

em que, Si é o percentual consumido da fonte de energia i em relação ao total. Foram utilizados o principal $[\mathrm{CR}(1)]$, os dois $[\mathrm{CR}(2)]$ e os $4[\mathrm{CR}(4)]$ maiores fontes energéticas.

O Índice de Herfindahl - Hirschman $(H H I)$, Equação 3, foi reconhecida por Hirschman (1964) em que foi calculado por meio do somatório do quadrado do Si de i até $\mathrm{p}$ fontes energéticas do SBOI.

$$
H H I=\sum_{i=1}^{p} S_{i}^{2}
$$

em que, p é o número de fontes de energia; Si é a participação da fonte energética i do consumo total da SBOI.

$\mathrm{O} \mathrm{HHI}$ encontra-se entre $1 / n$ (limite inferior) e 1 . Porém, quando as quantidades de fontes participantes variam ao longo do tempo, é necessário ajusta-lo para melhor 
comparação. Resende e Boff (2002) ajustaram o HHI conforme a Equação 4.

$$
H H I "=\frac{1}{p-1}(p \cdot H H I-1)
$$

A utilização dessa equação implica em um intervalo de variação entre 0 e 1 para o $H H I$. Feito este ajuste, o HHI" será avaliado entre 0 e 1 , sendo que 0 implica em condições de desconcentração e 1 implica em monopólio. A classificação é descrita pela tabela 1.

Tabela 1 - Classificação da concentração pelo HHl".

\begin{tabular}{lc}
\hline Classificação & Intervalo \\
\hline Desconcentrado & $0 \leq H H I^{\prime \prime} \leq 0,1$ \\
Pouco concentrado & $0,1 \leq H H I^{\prime \prime} \leq 0,18$ \\
Muito concentrado & $H H l^{\prime \prime}>0,18$ \\
\hline
\end{tabular}

Fonte: Resende e Boff (2002)

O Índice de Entropia de Theil (E), Equação 5, foi proposto Theil (1967).

$$
E=\sum_{i=1}^{p} S_{i} \cdot \ln \left(S_{i}\right)
$$

em que, $p$ é a quantidade de fontes de energia; $S_{i}$ é o matrix share, percentual, da fonte energética i do consumo total da SBOI.

Valores pequenos inferem em concentrações de energia mais elevadas. A quantidade de fontes de energia participantes é proporcional ao valor do $E$ que varia entre 0 , condição de monopólio, e $\ln (p)$, infere em participações iguais entre todas as fontes de energia (RESENDE; BOFF, 2002).

Analogamente ao HHI, é sugerido por Resende e Boff (2002) que para análises interanuais, a Entropia seja ajustada de acordo com Equação 6. E" apresenta como concentração máxima $\left(E^{\prime \prime}=0\right)$ e mínima concentração $\left(E^{\prime \prime}=1\right)$.

$$
E^{\prime \prime}=-\frac{1}{\ln (p)} \sum_{i=1}^{p} S_{i} \cdot \ln \left(S_{i}\right)
$$

O Coeficiente de Gini (G), Equação7, foi desenvolvido Gini (1955) em "Variabilità e mutabilità". Trata-se de uma medida de desigualdade, primariamente usada para avaliar desigualdade de renda, mas pode ser usada para mensurar a desigualdade do consumo de energia na SBOI. 


$$
G=1-\frac{\left[\sum_{i=1}^{p}\left(S_{i j}+S_{i}\right)\right]}{p}
$$

em que, $S_{i j}$ é a participação acumulada entre as fontes energéticas $i$ até $j ; S_{i}$ é participação percentual, da fonte energética i em relação ao consumo total do SBOI. Este índice está contido 0 e $1.0 \mathrm{G}$ classifica a desigualdade: nula a fraca $(0<G \leq 0,25)$, fraca a média $(0,25<G \leq 0,5)$, média a forte $(0,5<G \leq 0,7)$, forte muito forte $(0,7<G \leq$ $0,9)$ e muito forte a absoluta $(0,9<G \leq 1)$.

\section{RESULTADOS E DISCUSSÃO}

A Tabela 2 apresenta a evolução do consumo energético do Setor Brasileiro de Outras Indústrias (SBOI), em 10³ tonelada equivalente de petróleo (tep), de 1970 a 2016. Este segmento foi composto por 12 fontes na matriz energética. Em 2016, o SBOI representou $9,02 \%$ da demanda interna da energia industrial, e 3,15\% do consumo energético nacional. O SBOI consumiu, em 1970, 1,67 milhões tep e, em 2016, este valor chegou a 7,59 milhões tep, apresentando um crescimento médio de 3,35\% a.a. no período estudado. Este aumento do consumo de energia acompanhou o crescimento econômico das empresas que compõem o segmento.

Tabela 2 - Evolução do consumo energético do Setor Brasileiro de Outras Indústrias

(SBOI), em $10^{3}$ tonelada equivalente de petróleo (tep), de 1970 a 2016.

\begin{tabular}{l|cccccccccc}
\hline Fontes & 1970 & 1975 & 1980 & 1985 & 1990 & 1995 & 2000 & 2005 & 2010 & 2016 \\
\hline Gás natural & - & - & - & 27 & 262 & 343 & 567 & 984 & 1.901 & 2.183 \\
Carvão vapor & - & - & 8 & 25 & 9 & 6 & 152 & 99 & 87 & 11 \\
Lenha & 541 & 480 & 444 & 837 & 697 & 594 & 576 & 703 & 874 & 819 \\
Bagaço de cana & - & - & - & 2 & 4 & 9 & - & - & - & - \\
Óleo diesel & 127 & 127 & 114 & 58 & 120 & 159 & 150 & 113 & 144 & 120 \\
Óleo combustível & 644 & 1.636 & 2.211 & 799 & 835 & 1.031 & 803 & 358 & 177 & 56 \\
Gás liq. de petr. & 31 & 77 & 103 & 71 & 54 & 94 & 179 & 148 & 153 & 166 \\
Querosene & 16 & 56 & 68 & 43 & 25 & 7 & 5 & 5 & 1 & - \\
Gás canalizado & 4 & 9 & 27 & 40 & 22 & 2 & - & - & - & - \\
Eletricidade & 306 & 561 & 1.161 & 1.520 & 1.674 & 1.939 & 2.534 & 3.024 & 3.380 & 3.851 \\
Carvão vegetal & - & - & 8 & 29 & 16 & 13 & 8 & 10 & 12 & 12 \\
Outras secundarias & - & 3 & 227 & 4 & 2 & 15 & 374 & 380 & 481 & 376 \\
de petróleo & 1.670 & 2.948 & 4.371 & 3.455 & 3.720 & 4.213 & 5.347 & 5.823 & 7.211 & 7.592 \\
\hline Total & \multicolumn{7}{c}{ Fonte: EPE (2017). } \\
\hline
\end{tabular}

Em 1984 ocorreram os primeiros registros do Gás Natural na matriz energética do SBOI com 9 mil tep $(0,3 \%)$. O estímulo do consumo do gás natural no setor industrial 
ocorreu com a implantação do gasoduto Brasil-Bolívia, na década de 90 (EPE, 2014). Em 1995, o SBOI consumiu 343 mil tep de Gás Natural enquanto que, em 2016, foram 2,18 milhões tep, chegando com $28,75 \%$ do total do segmento. A TGC neste período (1984 a 2016) foi $18,81 \%$ a.a.

O setor vidreiro foi um dos precursores no consumo de Gás Natural do SBOI e é o principal consumidor quando comparado aos demais segmentos de outras Indústrias. Este combustível substituiu o óleo combustível, oferecendo vantagens técnicas para o melhor controle da temperatura na produção do vidro. Houve redução nos custos no sistema produtivo fabril pela logística por não possuírem contaminantes, aumentando o tempo entre as manutenções dos equipamentos (BAJAY; LEITE; GORLA, 2010a; MELO; MEDINA, 2005).

O consumo do Óleo Combustível caiu de 644 mil tep (1970) para 56 mil tep (2014). Teve crescimento de 1970 até 1980, onde atingiu o pico de demanda com 2,2 milhões tep, correspondendo a 56,8\% do consumo total do SBOI, e depois caiu até 2014 chegando a 59 mil tep. Esta queda foi resultado da Crise do Petróleo de 1979 que, de acordo com Pedrosa Junior e Corrêa (2016), elevou o preço do barril de petróleo e afetou severamente o consumo de Óleo Combustível por parte do SBOI.

A partir de 1983, a Eletricidade foi a principal fonte do SBOI. Apresentou um consumo estável, em que saiu de 1970 (468 mil tep) para 2016 (3,85 milhões tep, $50,72 \%$ do consumo total), com um crescimento médio de $5,66 \%$ a.a. Bajay, Leite e Gorla (2010b) afirmaram que o principal segmento consumidor de Eletricidade do SBOI é o não energo-intensivo e as principais finalidades para o uso da Eletricidade se distribui em $20 \%$ para aquecimento direto e $60 \%$ para execução de força eletromotriz.

A Lenha foi outra fonte de destaque no SBOI, que em 1970 teve consumo de 541 mil tep e 1981 caiu para 486 mil tep. Porém, a Crise de 1979 estimulou a demanda por lenha como reflexo de substituição do Óleo combustível. De 1982 até 1986, saiu de 605 mil tep para 961 mil tep, respectivamente. De 1987 (914 mil tep) até 2016 (819 mil tep) houve queda no consumo da Lenha, mas teve sua importância, pois contemplou em $10,78 \%$ da matriz energética do SBOI. As demais fontes não apresentaram boa representatividade.

A Figura 1 apresenta a evolução dos indicadores de concentração energética do setor de outras indústrias brasileira (SBOI), período de 1970 a 2016. A Figura 1.a mostra o comportamento da razão de concentração [CR(q)] do consumo energético do SBOI, no período de 1970 a 2016. De 1970 até 2016, a razão de concentração da principal fonte [CR(1)] ficou entre 56,78\% (1976) e 38,47\% (1981) com média de 47,59\%, inferindo que é altamente concentrado. As fontes dominantes no processo de obtenção energética no setor outras indústrias foram óleo combustível (1970 - 1982) e a eletricidade (1983 - 2016). O CR(1) cresceu de 38,58\% (1970) para 56,78\% (1976) e com a Crise de 79, o $\mathrm{CR}(1)$ caiu, atingindo seu valor mais baixo em 1981 e a partir de 1982 cresceu gradativamente de $38,60 \%$ para $50,72 \%$ (2016) da matriz energética do 
SBOI, mostrando-se assim altamente dependente da principal fonte energética.

De 1970 até 2016, a razão de concentração das duas principais fontes [CR(2)] saiu de $70,98 \%$ chegando ao valor máximo de $79,48 \%$ e teve valor mínimo de $61,25 \%$ (2001), proporcionando média de 70,53\%. A segunda principal fonte do SBOI foi a lenha (1970 - 1972 e 1984 - 1986), eletricidade (1973 - 1982), óleo combustível (1983 e 1987 - 2001) e gás natural (2002 - 2016). De 1970 a 1976, o CR(2) apresentou tendências de crescimento chegando 74,72\%. Entre 1977 e 1981 não houve muitas variações e a partir de 1982 teve crescimento gradativo saindo de 73,15\% para 79,48\%, em 2016.

Figura 1 - Evolução dos indicadores de concentração energética do setor de outras indústrias brasileira (SBOI), no período de 1970 a 2016. Sendo que (a) Razão concentração; (b) Índice Herfindahl-Hirschman; (c) Índice de entropia de Theil e; (d) Índice de Gini.
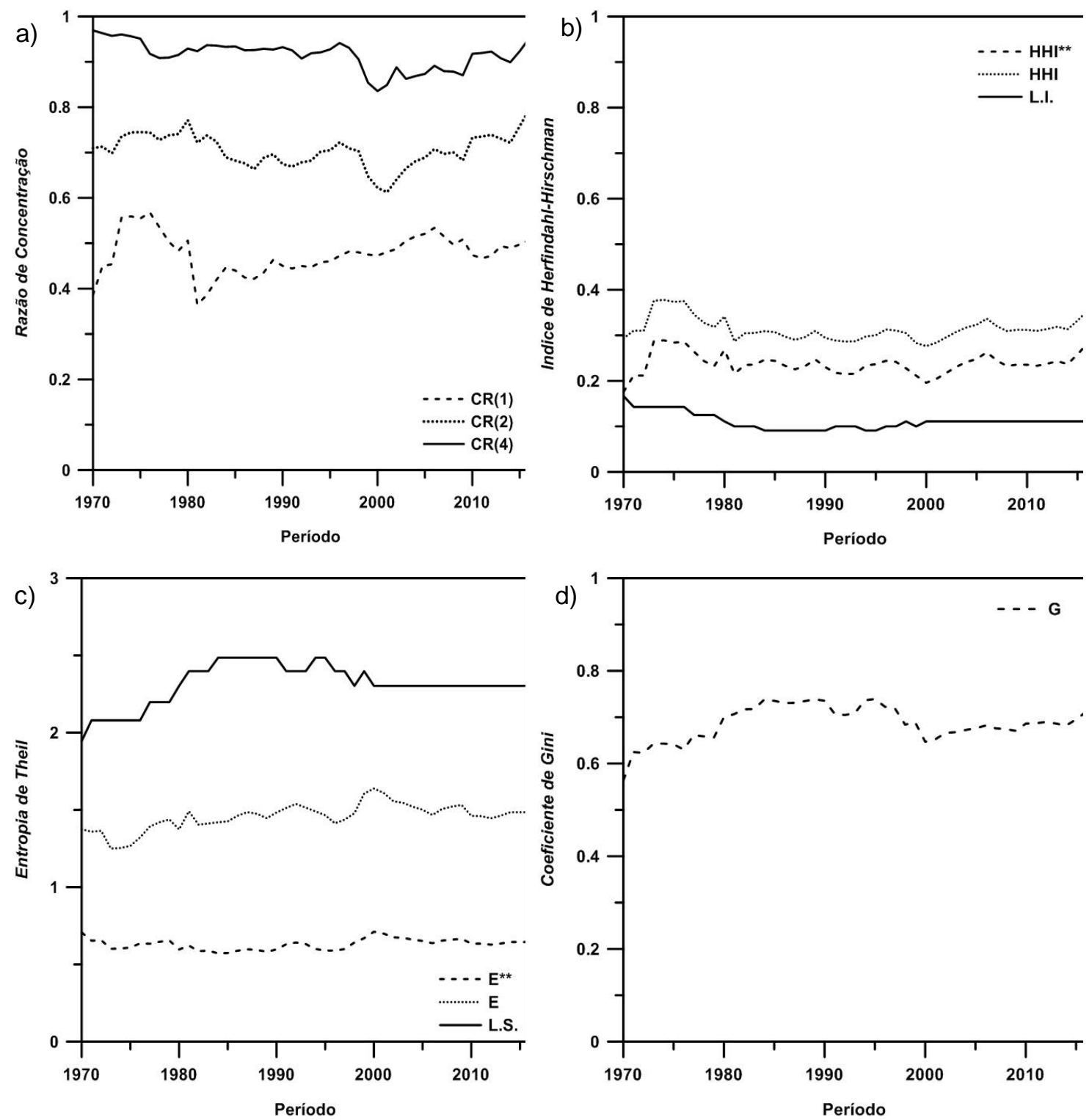
De 1970 até 2016, a razão de concentração das quatro principais fontes [CR(4)] variou entre 96,0\% (1970) e 83,56\% (2000), chegando 2016 com 95,21\%, proporcionando uma média de $91,51 \%$. Segundo a classificação de Bain (1959), a matriz energética da SBOI foi altamente concentrada nas quatro principais fontes, no período analisado. A terceira fonte energética do SBOI foi lenha (1973 - 1983,1987 2000 e 2003 - 2016), eletricidade (1970 - 1972), óleo combustível (1984 - 1986 e 2002) e gás natural (2001). E a quarta fonte foi a lenha (2001 e 2002), óleo diesel (1970 $1975,1981,1982)$, querosene (1982), gás natural (1987 - 2000), gás liquefeito de petróleo (1984 - 1986) e outras secundárias do petróleo (1976 - 1980 e 2003 - 2016).

A Figura 1.b apresenta a evolução do Índice de Herfindahl-Hirshman (HHI) do consumo energético do SBOI, no período de 1970 a 2016. O HHI" e as diferenças entre - $\mathrm{HHI}$ e o limite inferior mostraram que durante todo período, o consumo ficou classificado como muito concentrado. Observando evolução do HHl" em destaque de 3 períodos: 1970-1977; 1978-2001e 2002-2016. De 1970 até 1977, o HHI" teve tendências de crescimento com oscilações que saiu de 0,1760 para 0,2638 , em que sua classificação foi pouco concentrada para muito concentrada. De 1978 a 2001, o valor do HHI" caiu de 0,2431 para 0,2043 permanecendo seu grau de concentração. De 2002 a 2016, o HHI" teve tendências de crescimento saindo de 0,2178 para 0,2831, devido ao alto consumo de Eletricidade. A diferença entre HHI e o limite superior, entre 1970 a 1980 aumentou, elevando a concentração na matriz energética do SBOI. Após 1981 até 2000, a linha do $\mathrm{HHI}$ e do limite superior diminuiu, e diminuiu a concentração do consumo. De 2001 até 2014, voltou a crescer, sendo coerente com o comportamento do HHI".

A Figura 1.c apresenta a evolução da Entropia de Theil da SBOI, no período de 1970 a 2016. O índice de Entropia de Theil ajustada (E"), no período analisado, apresentou valores mínimo de 0,5714 (1984) e máximo de 0,7116 (2000), classificando com uma concentração alta. De 1970 até 1984 o E" decresceu, com oscilações, em que saiu de 0,6613 (1970) para o mínimo de 0,5714 (1984). A diferença entre o limite superior e E teve um aumento de 0,5708 (1970) para 1,0651 (1984), confirmado pelo E". De 1985 até 2000, houve um aumento expressivo, o E" saiu de 0,5739 para o pico, respectivamente. A diferença entre o limite superior e E cresceu de 1,0589 (1985) para 0,6641 (2000). Esta diminuição da concentração foi consequência da forte inserção do consumo do Gás Natural na matriz energética do SBOI. De 2001 até 2016, ocorreu uma diminuição no E", saiu de 0,6993 para 0,6449, respectivamente. Enquanto a diferença entre o limite superior e E aumentou de 0,6924 (2001) para 0,8177 (2016). Ocorreu um aumento da concentração, devido à queda do consumo do Gás Natural e aumento do consumo de Eletricidade, enquanto as demais fontes mantiveram o consumo absoluto quase constante.

A Figura 1.d representa a evolução do Coeficiente de Gini (G) da SIOB, no período de 1970 a 2016. Ao longo do período estudado o $G$ variou entre os intervalos: 0,5632 (1970) e 0,7393 (1995). A classificação da concentração ficou entre média a forte (1970 
- 1979, 1998 - 2015) e muito forte (1980 - 1997 e 2016). De 1970 até 1995 o G tendeu a crescer, saindo do mínimo até o máximo, mudando de classificação da desigualdade média a forte para forte a muito forte, entre 1979 e 1980. Este crescimento do G, bem como a mudança da desigualdade foi decorrente do aumento de fontes participantes, passando de 7 fontes (1970) para 12 (1995). De 1996 até 2016 o G decresceu, com oscilações, saiu de 0,7225 para 0,7116 , respectivamente. Variou a classificação da desigualdade de forte a muito forte para médio a forte entre os anos de 1997/1998 e 2015/2016. A queda na desigualdade é condizente com a queda do número de fontes participantes da matriz energética, em 1996 tinham-se 11 fontes participantes, já em 201610 fontes compuseram a matriz energética.

\section{CONCLUSÃO}

O consumo energético do SBOI teve um crescimento médio anual de 3,59\% a.a.. De 1970 até 1982, o Óleo Combustível teve como principal fonte a energética e foi substituída pela eletricidade a partir de 1983, devido ao tamanho das firmas e a disponibilidade de outras fontes de energia. A demanda por gás natural (1995 a 2016) cresceu $5,66 \%$ a.a., principalmente, em função dos incentivos no segmento vidreiro. $O$ $\mathrm{CR}(\mathrm{p})$ mostrou-se altamente concentrado por meio dos valores médios de $\mathrm{CR}(1)$ de $47,69 \%, \mathrm{CR}(2)$ de $70,54 \%$ e $\mathrm{CR}(4)$ de $91,51 \%$. O HHI inferiu como muito concentrado e o E corroborou com essas análises. O G mostrou tendência de concentração com desigualdade médio a forte para muito forte. O SBOI é concentrado na sua demanda energética.

\section{REFERÊNCIAS}

BAIN, J. Industrial Organization. New York: J. Wiley, 1959. 274 p.

\section{BAJAY, S. V.; LEITE, A. A. F.; GORLA, F. D. Oportunidades de Eficiência para} Indústria: Relatório Setorial: Setor Vidreiro. Brasília: CNI, 2010a. 56 p. (Relatório 16). Disponível em: http://www.procelinfo.com.br/services/DocumentManagement/FileDownload.EZTSvc.a sp?DocumentID $=\{B A E 14851-2 D 4 F-416 F-8840-$

D29251309AE7 $\} \&$ ServicelnstUID $=\{5 E 202 C 83-F 05 D-4280-9004-3 D 59 B 20 B E A 4 F\}$. Acesso em: 09 set. 2017.

BAJAY, S. V.; LEITE, Á. A. F.; GORLA, F. D. Oportunidades de Eficiência Energética para a Indústria: Relatório Setorial: não energo-intensivas. Brasília: CNI, 2010b. 53 p. (Relatório 11). Disponível em: http://www.procelinfo.com.br/services/procelinfo/Simuladores/DownloadSimulator.asp?DocumentID=\%7BAE3F378F\%2DC4C5\%2 D43B9\%2DAAB4\%2D1438B43DB70C\%7D\&ServicelnstUID=\%7B5E202C83\%2DF05 D\%2D4280\%2D9004\%2D3D59B20BEA4F\%7D. Acesso em: 18 jul. 2016.

BEIRAL, P. R. S.; MORAES, M. A. F. D.; BACCHI, M. R. P. Concentração e poder de mercado na distribuição de etanol combustível: análise sob a ótica da nova organização industrial empírica. Econ. Apl., v. 17, n. 2, p. 251-274, 2013. Disponível 
em: http://dx.doi.org/10.1590/S1413-80502013000200004. Acesso em: 09 set. 2017.

COELHO JUNIOR, L. M. Concentração regional do valor bruto de produção do pinhão no Paraná. Ciência Florestal, v. 26, n. 3, p. 853-861, 2016. Disponível em:

http://dx.doi.org/10.5902/1980509824213. Doi: 10.5902/1980509824213. Acesso em: 09 set. 2017.

COELHO JUNIOR, L. M. Concentração regional do valor bruto da produção do abacaxi no Paraná. RAMA - Revista em Agronegócio e Meio Ambiente, v. 10, p. 1015, 2017. Disponível em: http://dx.doi.org/10.17765/2176-9168.2017v10n4p10151028. Acesso em: 09 set. 2017.

COELHO JUNIOR, L. M. Concentração regional do valor bruto de produção da banana do Paraná, Brasil (1995 a 2010). Ciência Rural, v. 43, p. 2304-2310, 2013. Disponível em: http://dx.doi.org/10.1590/S0103-84782013005000137. Acesso em: 09 set. 2017.

COELHO JUNIOR, L. M. et al. Análise da concentração energética na indústria química brasileira. Enciclopédia Biosfera, v. 14, p. 1332-1341, 2017. Disponível em: http://dx.doi.org/10.18677/EnciBio_2017B111. Acesso em: 09 set. 2017.

COELHO JUNIOR, L. M. et al. Concentração da demanda energética da indústria brasileira de alimentos e bebidas. Enciclopédia Biosfera, v. 27, p. 1, 2018. Disponível em: http://dx.doi.org/10.18677/EnciBio_2018A114. Acesso em: 09 set. 2017.

COELHO JUNIOR, L. M.; REZENDE, J. L. P.; OLIVEIRA, A. D. Concentração das exportações mundiais de produtos florestais. Ciência Florestal, v. 23, n. 4, 2013. Disponível em: http://dx.doi.org/10.5902/1980509812353. Acesso em: 09 set. 2017.

COELHO JUNIOR, L. M.; SANTOS JUNIOR, E. P.; ALENCAR, F. V. Concentração da demanda energética na Indústria Brasileira Têxtil. Engevista (UFF), v. 21, p. 79-92, 2019. Disponível em: https://doi.org/10.22409/engevista.v21i1.13190. Acesso em: 09 set. 2017.

COSTA, N. L.; SANTANA, A. C. Estudo da concentração de mercado ao longo da cadeia produtiva da soja no Brasil. Revista de Estudos Sociais, v. 16, n. 32, p. 111135, 2014. Disponível em: http://dx.doi.org/10.19093/res.v16i32.1853. Acesso em: 09 set. 2017.

CUENCA, M. A. G.; DOMPIERI, M. H. G. Dinâmica espacial da canavicultura e análise dos efeitos sobre o valor bruto da produção, na região dos tabuleiros costeiros da Paraíba, Pernambuco e Alagoas. Revista Econômica do Nordeste, v. 47, n. 4, p. 91106, 2017. Disponível em: https://ren.emnuvens.com.br/ren/article/view/620/497. Acesso em: 20 de jun. 2017.

EPE - EMPRESA DE PESQUISA ENERGÉTICA. Balanço Energético Nacional 2017, Base 2016. Disponível em: https://ben.epe.gov.br/. Acesso: 20 jun. 2017.

EMPRESA DE PESQUISA ENERGÉTICA - EPE. Nota Técnica DEA 13/14: Demanda de Energia 2050. Rio de Janeiro: EPE, 2014. Disponível em:

http://www.epe.gov.br/Estudos/Documents/DEA\%201314\%20Demanda\%20de\%20Energia\%202050.pdf. Acesso em: 02 de out. 2017.

GINI, C. Variabilità e mutabilità. In: PIZETTI, E.; SALVEMINI, T. (ed.). Reprinted in memorie di metodologica statistica. Rome: Libreria Eredi Virgilio Veschi, 1955. 
HIRSCHMAN, A. O. The paternity of an index. The American Economic Review, v. 54, n. 5, p. 761-762, 1964.

LUFT, A.; ZILLI, J. B. Concentração de mercado: uma análise para a oferta de crédito pelo setor bancário brasileiro. Revista Teoria e Evidência Econômica, v. 19, n. 41, 2013. Disponível em: http://dx.doi.org/10.5335/rtee.v0i41.3737. Acesso em: 09 set. 2017.

MELO, M. S. M.; MEDINA, P. L. N. Gás Natural: uma análise econômica da demanda focada no setor industrial. Rio de Janeiro: UFRJ, 2005. Disponível em:

http://www.anp.gov.br/CapitalHumano/Arquivos/PRH21/Marcelo-Silva-de-Matos-Meloe-Pedro-Leonardo-Neves-Medina_PRH21_UFRJ_G.pdf. Acesso em: 20 jul. 2016.

OLIVEIRA, J. C.; SPERB, A. F. N. Análise da evolução da concentração na indústria siderúrgica brasileira entre os anos de 1991 e 2013. Estudos do CEPE, n. 43, p. 84100, 2016. Disponível em: http://dx.doi.org/10.17058/cepe.v0i43.7626. Acesso em: 09 set. 2017.

PEDROSA JUNIOR, O. A.; CORREAA, A. C. F. A crise do petróleo e os desafios do présal. Boletim de Conjuntura, n. 2, p. 4-14, 2016. Disponível em:

http://www.fgv.br/fgvenergia/oswaldo_pedrosa_petroleo/files/assets/common/download s/publication.pdf. Acesso em: 22 maio 2017.

POSSAS, M. L. Estruturas de mercado em oligopólio: economia e planejamento. 2. ed. São Paulo: Hucitec, 1999. 191 p.

RESENDE, M.; BOFF, H. Concentração industrial. In: KUPFER, D.; HASENCLEVER, L. (Org.). Economia industrial: fundamentos teóricos e práticas no Brasil. Rio de Janeiro: Campus, 2002. p. 73-90.

SANTANA, P. H. M.; BAJAY, S. V. New approaches for improving energy efficiency in the Brazilian industry. Energy Reports, v. 2, p. 62-66, 2016. Disponível:

https://doi.org/10.1016/j.egyr.2016.02.001. doi: 10.1016/j.egyr.2016.02.001. Acesso em: 09 set. 2017.

SIMIONI, F. J. et al. Evolução e concentração da produção de lenha e carvão vegetal da silvicultura no Brasil. Ciência Florestal, v. 27, n. 2, p. 731-742, 2017. Disponível em: http://dx.doi.org/10.5902/1980509827758. Acesso em: 09 set. 2017.

THEIL, H. Economics and information theory. Amsterdam: North-Holland, 1967. $488 p$. 